\title{
Jardín, de Dulce María Loynaz: de la obra a la crítica social
}

Jardín, by Dulce María Loynaz: From the boOK to social CRITIQUe

Laura Carolina Castañeda-Sua*

* Universidad de Guadalajara, México

Correo-e: lccastanedas@unal.edu.co (D) https://orcid.org/-0001-0000 5927-6383 Recibido: 18 de julio de 2019 Aprobado: 28 de mayo de 2020

Resumen: Se analiza la novela Jardín (1935), de la poeta cubana Dulce María Loynaz, con el fin de demostrar el potencial de la literatura para cuestionar las relaciones sociales y de poder y, simultáneamente, proponer una comprensión de lo latinoamericano. Para ello se toman en cuenta tres elementos: la temporalidad, la relación naturaleza/género y la dicotomía barbarie/civilización. Se hace énfasis tanto en el contexto de producción de la obra como en el texto en sí mismo (desde el punto de vista discursivo) para explicar los aportes específicos de la novela a la discusión social y literaria del momento.

Palabras clave: literatura latinoamericana; novela; análisis literario; ciencias sociales y humanas: mujer; civilización; modernización; poder político

Abstract: Cuban poet Dulce María Loynaz's novel Jardín [Garden] (1935) is analyzed with a view to showing the potential of literature to contest social and power relations and proposing a comprehension of the Latin American at once. To do so, three elements are taken into account: temporariness, the nature / gender relation and the barbarism / civilization dichotomy. Both the book's writing context and the text itself (from the discursive standpoint) are highlighted in order to explain the specific contributions of the novel to the present social and literary discussions.

Keywords: Latin American literature; novels; literary analysis; social and human sciences; women; civilization; modernization; political power 


\section{INTRODUCCIÓN}

Se presenta un análisis sociopolítico de la novela Jardín (1935), escrita por la poeta cubana Dulce María Loynaz. Al proponer un estudio de este tipo, buscamos subrayar el potencial de la obra para problematizar las relaciones de poder de una sociedad en un tiempo y lugar concretos, en este caso, América Latina y el Caribe. Tal apuesta metodológica implica liberar a la literatura de su condición de "formulación retórica o expresión sensible" (Linares, 2011: 34) para dar paso a su comprensión como forma de enunciar las relaciones de exclusión, dominación o resistencia de las sociedades, lo cual denota su importancia para entender ciertas tensiones y conflictos.

Se reflexiona frente a los ámbitos de producción, mediación y recepción de la novela de Loynaz. Tratamos de comprender: a) el contexto en el cual emerge la obra; b) el texto en sí mismo, es decir, la lectura específica sobre la realidad que propone, lo cual incluye los estereotipos y percepciones inscritos en la novela (Hall, 2006); y finalmente, c) las diferentes interpretaciones que se han hecho sobre Jardin y sus implicaciones en la literatura latinoamericana. Se pone así de manifiesto la centralidad del arte en la reproducción de la sociedad, rompiendo su percepción como expresión subjetiva e individual.

Cabe anotar que nuestra propuesta retoma a la poeta cubana, puesto que, a pesar de la existencia de estudios sobre su obra, éstos no agotan las posibilidades analíticas y aportes críticos de la misma. Lo anterior pese a que Loynaz es considerada una de las figuras más sobresalientes dentro de la literatura de la isla, a la altura de José Lezama Lima y Alejo Carpentier (Caballero, 2005). A su vez, Jardín es la única novela de la autora, con publicación tardía tras su escritura, pues a pesar de ser terminada en 1935 salió a la luz hasta 1951, y únicamente se reeditó en 1992 (Caballero, 2005: 6). Esta situación suscita distintas interrogantes sobre la difusión e interpretación del texto, así como de su lugar en las letras latinoamericanas y el papel que podría ocupar la condición de mujer de Loynaz en relación con su producción artística.

Jardín es, de este modo, un caso que podría considerarse atípico y merece ser estudiado con detenimiento para determinar su lugar en el marco de la literatura de su época, así como para comprender sus diferencias, similitudes y aportes específicos al arte y la crítica social. Como hemos insistido, la novelística tiene un rol activo en la construcción de los imaginarios, percepciones (Hall, 2006) y prejuicios a partir de los cuales entendemos la realidad.

\section{LOYNAZ FRENTE A LAS PRODUCCIONES DE SU ÉPOCA}

La obra de Dulce María Loynaz se produce en un momento crucial para la narrativa latinoamericana. Durante las primeras décadas del siglo XX, la literatura en nuestra región sufre un giro y busca deslindarse del canon europeo para salir en busca de su identidad (Polo García, 1982).

Definir qué somos y cuál es nuestra particularidad respecto al Viejo Continente será el propósito de las producciones literarias en este periodo (Millares, 1993). Durante los años veinte y treinta de dicha centuria emerge lo que algunos denominan la novela de la tierra o telúrica (Márquez Rodríguez, 2002). Este tipo de obras tiene la particularidad de que, a la par de realizar una búsqueda de la identidad de lo latinoamericano, recurre a la constante mención de la naturaleza. El llano, la pampa y la selva serán sus protagonistas y se volverán el puente para abordar la dicotomía barbarie/civilización (Fernández López, 2000), eje sobre el que versarán muchas de las novelas de la época.

La representación de la naturaleza como un 'personaje/presencia' (Oyarzún, 2000: 26) se convierte en la temática definitoria de la región. En palabras de Polo García, la llamada novela de la tierra se "convirtió en la expresión 
latinoamericana por excelencia” (1982: 23), la manifestación por antonomasia del 'nosotros', marca particular de nuestras obras.

Numerosos son los análisis sobre este periodo de producción de las letras del continente. Sin embargo, es importante resaltar la ausencia de estudios respecto a la relación entre la naturaleza y el género ${ }^{1}$ en dichas obras. Esto quiere decir que, aunque desde la década de los ochenta existen investigaciones dedicadas a analizar el género en la literatura latinoamericana y una parte de ellas se enfoca en la naturaleza y lo femenino, son pocas las que se dedican a establecer su papel específico en las novelas de inicios del siglo XX caracterizadas como telúricas o de la tierra.

Explorar dicha relación es importante, puesto que las novelas de esta época no sólo recurren a la naturaleza para desarrollar la trama, sino que su presencia es acompañada por un uso sistemático de cualidades femeninas y masculinas. La presencia de estas particularidades remarca los rasgos de la barbarie frente a los de la civilización, pues la naturaleza es exaltada como el distintivo del salvajismo por excelencia en esta parte del mundo. Lo anterior indica que, junto con el género, la naturaleza es central para establecer una diferencia entre lo bárbaro y lo civilizado e introducir relaciones de dominación, control y explotación en función de lograr la transformación del abrumador ambiente latinoamericano para dar paso al orden y al progreso.

Ahora bien, en el marco de este rasgo inexplorado por la crítica literaria convencional se inscribe la importancia de Jardín. La obra de Loynaz cumple con la caracterización general de la época: la naturaleza como personaje/presencia,

1 Retomamos lo masculino y lo femenino, puesto que la novela nos propone esta visión binaria a la hora de describir la naturaleza. Con esto no desconocemos que el género se presenta de más maneras, sólo consideramos lo que la propia obra nos brinda para realizar el análisis, evitando superponer categorías que no existen dentro de la misma. Como denota Lugones (2005), recurrir a una clasificación, en nuestro caso la binariedad, no implica desconocer la complejidad del género, por el contrario, permite entender cómo opera el pensamiento binario y lo visibiliza a la vez que lo denuncia. su protagonismo sobre lo humano y la dicotomía civilización/barbarie. No obstante, su exploración del género difiere de la que realizan otros autores contemporáneos.

Frente a La vorágine (1924), de José Eustasio Rivera; Don Segundo Sombra (1926), de Ricardo Güiraldes; Doña Bárbara (1929), de Rómulo Gallegos; Raza de bronce (1919), de Alcides Arguedas; Canaán (1902), de José Pereira da Graça Aranha; El gaucho florido (1932), de Carlos Reyles; Los de abajo (1916), de Mariano Azuela; y El mundo es ancho y ajeno (1941), de Ciro Alegría (Fernández López, 2000); Jardín propone una descripción contingente del género (Caballero, 2005) en donde lo masculino y lo femenino se asocian a la naturaleza para denotar diferentes relaciones de dominación o resistencia que ponen en tela de juicio el vínculo naturalezafeminidad que se presenta en el conjunto de las obras del periodo.

De esta manera, puede decirse que Jardín, a pesar de ser producto de su época, va más allá de las apuestas de sus contemporáneos. En contraste con otras novelas, que se dedican a describir el medio con características femeninas, Jardín hace uso de rasgos de ambos géneros para dotar de múltiples sentidos al paisaje y, por este camino, complejizar nuestra región y la presencia de la naturaleza como atributo especifico de la misma.

Lo anterior no debe considerarse un rasgo singular, fruto de la genialidad única de la escritora y objeto de análisis estético, sino que puede ser entendido como una disputa dentro del campo literario. La obra de Loynaz usa elementos comunes a las novelas de su tiempo, es decir, la naturaleza, lo femenino y lo masculino, dándoles matices diferentes con el fin de ofrecer una comprensión particular sobre la realidad de aquel momento, en donde lo masculino se asociaba a la ciudad y lo femenino a la naturaleza.

Si el conjunto de la literatura en que se inscribe el trabajo de la poeta cubana estuvo preocupado por definir la identidad mediante la dicotomía barbarie/civilización y el uso de la naturaleza con 
características de género marcadas, es claro que la particular manera de relatar de Loynaz quiere expresar una alternativa para entender el 'nosotros' latinoamericano.

La serie de atributos que ya hemos denotado es empleada por la escritora de forma no convencional. La poeta cubana da giros a los elementos que le brinda su contexto literario para reconfigurar su significado (Caballero, 2005) a lo largo de la trama y, de esta manera, disputar los marcos de interpretación que nos dan unidad como miembros de una región.

Así las cosas, la literatura juega un rol activo en la configuración de lo social y lo político, además, esta obra en particular y su autora denotan que este papel es heterogéneo. No hay una sola manera de establecer un nosotros y, por tanto, esa identidad se construye desde diferentes ángulos y voces. Jardín cuestiona el campo literario en el que se inscribe y propone alternativas que sobrepasan sus límites.

En el siguiente apartado se muestra cuáles son los matices específicos que la obra, heterodoxa y poco leída en relación con sus pares, aporta a la narrativa de la época.

\section{LA LECTURA MÁS ALLÁ DE SU AUTORA}

Para poder abordar una lectura de la obra como la que proponemos, es necesario realizar algunas aclaraciones analíticas. En primer lugar, partimos del hecho de que la novela debe considerarse como un texto que disputa los significados desde su propia narrativa y, a su vez, entreteje múltiples dimensiones sociales (Barthes, 1984) que sobrepasan las intenciones del autor.

En ese sentido, no buscamos centrarnos en el valor artístico o estilístico de Jardín, sino explorar las diferentes formas en que participa en los debates de su tiempo y en si tiene vigencia dentro de la realidad latinoamericana contemporánea.
También vale aclarar en este punto que no se trata de una novela autobiográfica. Como lo declara su propia autora en conversaciones con Aldo Malo, "se ha querido ver en Jardín un libro autobiográfico, lo cual no es cierto [...] creo ser una criatura humana, más interesante que Bárbara, que al fin y al cabo no es más que una mujer como otra cualquiera" (Loynaz, en Martínez Malo, 1999: 55). Lo anterior reafirma la necesidad de un análisis que sobrepase el entendimiento de la obra como un producto individual desligado del contexto social.

En segundo lugar, dada la complejidad de la novela, sólo abordamos algunos de los múltiples tópicos que ésta trata. Nos enfocamos en el tratamiento del tiempo, la dicotomía civilización/ barbarie y la relación entre naturaleza y género. Estos temas específicos permiten poner el acento en la configuración del nosotros latinoamericano, punto clave para entender la importancia de la obra en el ámbito social.

\section{LA TEMPORALIDAD}

La forma en que se presenta el tiempo en Jardín es uno de los elementos que más se han analizado por la crítica. Lejos de ser lineal, cronológicamente ordenado, éste remite a los recuerdos, la nostalgia y el descubrimiento personal por medio de la memoria.

Bárbara, el personaje principal de la novela, remueve los objetos de sus antecesoras para hallarse. Como se relata, "quiso pensar, recordar. Procuró evocar todo lo que más la había conmovido en su vida; era preciso fijar sus sensaciones para poder encontrarse, necesitaba una emoción, una sola, para revelarse a sí misma" (50). ${ }^{2}$ Esta doble temporalidad conduce a que el presente sólo se constituya mediante el pasado. Dicha

2 Todas las citas pertenecientes a Jardín corresponden a Loynaz, 1992, por lo cual sólo se anota el número de página. 
comprensión relacional de la realidad se distancia del relato lógico cronológicamente ordenado de sus contemporáneos que podemos ver en obras como Doña Bárbara.

Esta característica no sólo es novedosa en términos de la narración, sino que muestra una crítica general a la secuencia pasado-presente, producto de la percepción de una modernidad que parece estar vigente en el contexto de la producción misma de la obra, como deja ver la ya mencionada oposición barbarie/civilización.

En este mismo sentido, Loynaz también muestra cómo en el jardín hay una suerte de tiempo detenido frente a la incontenible velocidad de la urbe. A Bárbara le parece "que desde el instante en que, después de pasear por el jardín, fue a sentarse, displicente y soñadora, a mirar retratos viejos, había transcurrido un tiempo que ya no podría contar por horas, sino por años" (43); por el contrario, en la ciudad "se trata por todos los medios de satisfacer, de apaciguar al menos, ese afán, esa premura extraña que ya va siendo como una demencia de la generación” (174).

La descripción del tiempo de la metrópoli con el adjetivo 'demencia', que hace referencia a una patología, denota otro cuestionamiento al progreso y su materialización en la ciudad y sus ritmos, esta vez un poco menos en términos relacionales, pero sí interpelando a la crítica de lo moderno y la civilización. Ahora bien, esto no está desligado de la condición de género que parece atravesar toda la obra. El último día de Bárbara en el jardín, antes de zarpar hacia la ciudad, piensa su viaje en los siguientes términos:

Mañana era él, nudo seguro de sus brazos, refugio cierto de su pecho; mañana era él, paz de sus ojos, bienandanza de su presencia. Mañana era lo sano por lo mórbido, lo real por lo absurdo, lo natural por lo torcido... iLo natural, lo natural sobre todo! Lo natural de todo él, bueno, armonioso, limpio (145).
Es necesario señalar cómo en este fragmento el porvenir es catalogado como "lo sano", lo "armonioso", "lo real". Esta serie de calificativos deseables a su vez se acompaña con la caracterización de lo masculino. La frase "mañana era él" hace referencia al marinero, por lo cual el futuro ansiado queda ligado a este género. El futuro es masculino e impera sobre esa naturaleza que parece aprisionarla.

Asimismo, es importante resaltar cómo Loynaz no se queda en esta descripción simple. La autora complejiza su argumentación pues, posteriormente, Bárbara encuentra que la luz eléctrica, el símbolo de su entrada en la civilización, "no puede mostrarle un mundo mejor que el que ya ella había poseído en soledad, sólo con la fuerza de su deseo" (163) y en su jardín. Esta es una vuelta a aquello que se había catalogado como anómalo y una matización del progreso masculino imperante como lo deseable.

Hasta el momento, hemos explicado cómo el corazón de la crítica de Dulce María Loynaz apunta hacia la complejidad de la división barbarie/civilización, por lo que pasaremos ahora a profundizar en este aspecto.

\section{Civilización y Barbarie, PRoblema ACTUAL}

La búsqueda de Bárbara por ingresar 'al mundo' es uno de los ejes conductores de la novela. La intención del personaje en los primeros capítulos es entenderse a sí misma y salir del medio natural que parece aprisionarla:

Es el jardín obscuro invadiendo la tierra. ¿No sientes el jardín minando los cimientos del mundo, el jardín que taladra el piso por donde andas, que levanta imperceptiblemente las alfombras de los palacios, las planchas de acero de las fábricas de la civilización? (41). 
En este punto de la narración, la naturaleza aparece como la oposición a la civilización añorada. No obstante, con el desarrollo de la trama y tras conocer múltiples ciudades y establecer una familia, Bárbara decide regresar a su jardín en los capítulos finales. Como mencionamos anteriormente, el mundo y sus artefactos finalmente quedan relegados en la percepción de la protagonista, quién concluye que éstos no resultan ser tan extraordinarios respecto a su jardín. El anhelo por encontrar la civilización, presente en las primeras páginas, es cuestionado. Ya no se trata de la magnificencia de la metrópoli frente a la naturaleza, sino de su desmitificación.

El regreso al jardín implica una crítica a ese progreso lineal en donde la ciudad y la técnica no pueden ser cuestionadas, pero también a la modernidad (Caballero, 2005) en tanto oposición a la naturaleza y finalidad última del sujeto.

Dicha crítica no se detiene en este punto, pues toda la obra de Dulce María Loynaz constituye un intento por desarticular la manía de clasificación que impone el mundo moderno: "La humanidad tiene miedo del vacío - abstraer es hacer vacío-, de las cosas que no puede definir, medir y tocar. Y lo que no toca ni mide le parece que está vacío" (85). La novela trata entonces de "humanizar el objeto y fusionar el sujeto con la naturaleza" (5), mediante una escritura en donde todo transita y no hay una caracterización fija, donde son múltiples las descripciones y van cambiando según el desarrollo de la trama.

La condición provisional y abierta de las definiciones es notable en el marco del tratamiento del género que realiza la autora. Al respecto, el siguiente apartado trata de dar luces sobre la fluidez de las caracterizaciones de la naturaleza y los sujetos.

La crítica a la modernidad que se retrata en la novela es actual y no únicamente una problematización literaria. Por el contrario, se trata de una cuestión por la que un gran segmento de los estudios sobre América Latina se ha enfrentado constantemente (Camejo Ochoa, 2014), lo cual deja entrever el potencial de la obra para aportar al análisis de la realidad.

\section{DE LA NATURALEZA Y EL GÉNERO}

A la crítica explícita que realiza Loynaz a las definiciones y su delimitación, se une el cuestionamiento implícito por la condición de género, el cual se asocia constantemente a la descripción de la naturaleza: el jardín y el mar.

Dentro de la novela, eljardín aparece caracterizado como "madre saturnal, la insaciable devoradora de sus propios hijos" (88). A la tradicional idea de la naturaleza como progenitora y lugar de vida se le da un matiz siniestro: "es la madre prolífera, fecunda, incansable, la madre perennemente horizontal recibiendo la simiente de la vida que son los muertos, pariendo los renuevos de la muerte que son los vivos" (88). Dicha adjetivación rebasa el carácter romántico común en textos contemporáneos.

Lo anterior no es un aporte novedoso que realiza Loynaz, pues entre las obras de su época es común encontrar el paso de la descripción idealizada de la naturaleza a la lúgubre (Magnarelli, 1996). No obstante, lo que sí puede considerarse una contribución suya es la fluctuación del género a lo largo de la trama, es decir cómo toma diferentes características más allá de la visión romántica y además se asocia tanto a lo femenino como a lo masculino.

El jardín siniestro relacionado con la madre se muestra posteriormente como "un peladero miserable, con miseria de viejo flaco e hipócrita" (72), cuya intención es aprisionar a Bárbara, es decir, toma una forma masculina decrepita que se aleja de la primera descripción.

De la misma forma, al final de la novela, nuevamente el jardín se transforma y vuelve a ser femenino, pero esta vez maternal y deseable. "Dormir, volver, reintegrarse al vientre tibio de la sombra sin nacer todavía, sin saber de las luces 


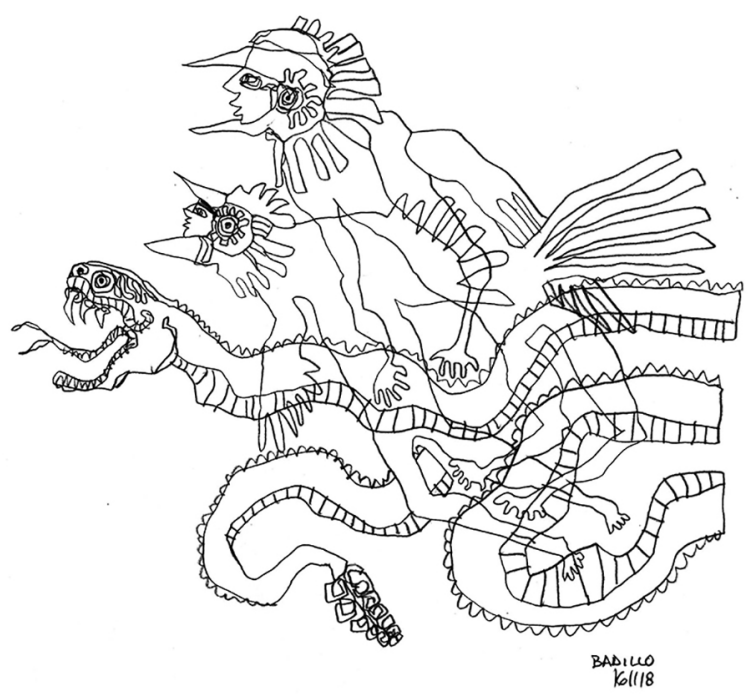

Quetzalcóatl simple, de la serie Dibujo simple (2018). Lápiz: Carlos Alberto Badillo-Cruz.

Prohibida su reproducción en obras derivadas.

de los hombres" (189) es la intención de Bárbara en su viaje de retorno, mostrando el jardín como refugio en contraposición a la prisión, inversamente a lo que ocurre en la primera parte de la obra. Lo femenino se vuelve grato y ansiado.

El devenir del género en términos masculinos también se presenta en esta narración. "El mundo, la velocidad, la razón, son lo masculino” (Caballero, 2005: 38) tanto como la civilización, a la que es llevada Bárbara por un personaje varón, el marino. Pero la caracterización dicotómica entre civilización masculina y naturaleza femenina se pone en entredicho, pues el mar también es objeto de humanización y se asocia a lo varonil:

Sólo el mar era bueno, y era grato ir a él como a un amigo viejo que escuchara sin cansarse una misma historia, siempre empezada y nunca terminada $[\ldots]$ (37).

La Niña es buena y lleva una medallita de oro para alejar al diablo. Nada ha de sucederle cuando vaya junto al mar o junto a los hombres (25).

No es gratuito que el personaje masculino llegue atravesando el mar. El lugar de los hombres, la ciudad y la seguridad sólo se alcanzan mediante este tránsito. Además, en esta ocasión la naturaleza no sólo se describe en términos femeninos, sino que retoma lo masculino para demarcar 'lo bueno', 'lo seguro', 'lo virtuoso'. El uso de este recurso muestra una diferencia entre la escritura de la cubana y sus contemporáneos, quienes al referirse a la naturaleza sólo recurren a elementos femeninos para describirla. ${ }^{3}$

En síntesis, podemos decir que la novela de Loynaz remite a una crítica a la dicotomía moderna, que se cimenta en el uso de la fluidez y la indeterminación. El género es un elemento transversal al tiempo, al debate civilización/barbarie y a la narración de la naturaleza. Las contradicciones en Jardín son simbióticas (Caballero, 2005: 26), esto es, relacionales, en tanto ninguna constituye la total oposición de la otra sino su continuación. La búsqueda de la civilización sólo desemboca en un regreso a la naturaleza. La feminidad siniestra del jardín da paso a lo maternal. Como lo muestra Caballero:

3 Al respecto, véase el trabajo de Sharon Magnarelli "La mujer y la naturaleza en La voragine: a imagen y semejanza del hombre" (1987). 
Bárbara había querido las luces de los hombres y ya no podía librarse de ellas; tampoco los hombres con tantas luces podrían librarse de la sombra que ella les llevaba, sombra de vientre femenino, de cólera divina de jardín anochecido (2005: 37).

A lo que invita la obra es a entender la capacidad constitutiva de las oposiciones en la realidad. Esta exhortación supera las apuestas literarias de la época que hacen uso de las características femeninas para remarcar la barbarie del continente frente a un porvenir civilizado con rasgos masculinos. Lo anterior pone de manifiesto el potencial de la novela para explorar una idea de lo latinoamericano que supera esa visión dicotómica de la modernidad, misma que, en el caso de nuestra región, se manifiesta en el afán de dominar la naturaleza y buscar la civilización.

Optar por una fluidez en la argumentación fue una apuesta, en ese entonces política, que sobrepasó la perspectiva del progreso lineal y la identidad latinoamericana uniforme. La novela, así, dialoga con la actualidad, pues la discusión sobre estos temas en nuestra región (Stavenhagen, 1981) sigue estando vigente en los círculos dedicados a las ciencias sociales.

\section{LA RECEPCIÓN DE LA OBRA DE LOYNAZ}

Hasta aquí hemos señalado el papel del género y los aportes de Jardin al debate sobre la modernidad. Como hemos dicho, la obra no permanece cerrada y se inserta en los debates contemporáneos, pues Loynaz, más que hacer una escritura para el deleite estético, irrumpe en los temas sociales medulares de nuestra América.

En este contexto, no sorprende que las lecturas de la novela en nuestros días se centren sobre todo en el feminismo (Smith, 1993) corriente de pensamiento que, supuestamente, aparece en esta obra. Nuestra intención frente a estos argumentos es mostrar que la escritura de la poeta cubana propone una visión compleja del género que no se reduce a integrar a la mujer en la narrativa. Con este propósito, explicaremos y discutiremos las interpretaciones que versan sobre el asunto.

Para Smith, "Jardín es la obra pionera del feminismo en la narrativa latinoamericana" (1993: 264), pues considera que la novela tiene como finalidad la búsqueda de la identidad femenina. Para ilustrar su posición, la autora nos indica que en el orden simbólico masculino la mujer no tiene cabida (Smith, 1993: 272), de allí que Bárbara nunca pueda verse en el espejo. Esta ausencia de representación "en el orden patriarcal” (Smith, 1993: 272) hace que la protagonista se busque a sí misma. Smith adapta el cuento de la Bella Durmiente, así como la travesía del personaje por mar, para simbolizar el paso de la mujer pasiva a activa que toma sus propias decisiones.

En esta misma línea de argumentación camina Blanca Aurora Mondragón Espinoza (2008), quien realiza todo un análisis dedicado al relato arriba citado para mostrar cómo la protagonista pasa a ser sujeto y no sólo objeto de decisiones y deseo. Mediante un estudio de la intertextualidad, Mondragón Espinoza afirma que Loynaz resignifica el cuento tradicional que nos mostraba una mujer siempre a la espera del hombre y su respuesta.

Tanto Mondragón Espinoza como Smith realizan estos aportes en clave de género sin perder de vista el papel crítico a la modernidad que se presenta en Jardín, de suerte que tenemos unas relecturas que destacan la búsqueda de lo femenino y buscan rescatar a la escritora latinoamericana y su contribución a la literatura de la región. Sin embargo, es interesante notar cómo la naturaleza no parece ser un eje en estos análisis. Si bien la crítica al rol de la mujer y la modernidad tiene fundamento, consideramos necesario resaltar que no está completa si se deja de lado dicha perspectiva. 
Para Verity Smith (1993), la naturaleza constituye uno de los puntos débiles de la obra de la cubana, pues gracias a su presencia se cae en un esencialismo que condena a la mujer a tener un papel inferior y "permanecer fuera de la historia” (Mondragón Espinoza, 2008: 275). No obstante, como hemos venido argumentando, posee una condición fluctuante que está muy lejos de responder a esta interpretación.

La naturaleza y su relación con el género es una potencialidad de la novela de Loynaz. El recorrido descriptivo en donde lo masculino y lo femenino se trasforman cuestiona el principio de categorización moderno donde todo debe ceñirse a una delimitación estricta. El vínculo feminidad-naturaleza no es unívoco e inmutable, como en las obras de su época, e invita a pensar en una desestructuración de ese patrón binario.

Loynaz habla entonces de una condición abierta que tienen tanto la naturaleza como la protagonista, pues Bárbara también cambia según su relación con los otros en el devenir de la trama. No encontramos simplemente la inclusión de la voz femenina, sino que presenciamos su desencialización mediante las múltiples caracterizaciones de la naturaleza y el género, lo que algunos llamarían polifonía (Caballero, 2005), y que permite no sólo ver a la mujer sino diferentes interpretaciones sobre ella.

Ahora bien, cabe anotar que la conceptualización de la mujer se presenta como un universal que olvida la heterogeneidad de dicha condición. Tal categoría pasa por alto las circunstancias de raza, clase, edad y nacionalidad (Lugones, 2005) que atraviesan los diferentes cuerpos. Tendríamos entonces que preguntarnos a quiénes exactamente se refieren estas relecturas.

En este punto es necesario resaltar que Bárbara no es el único personaje femenino de la novela. Laura, la sirvienta negra, es la otra mujer o, más bien, la mujer otra que aparece en el jardín. A pesar de que la obra permite la emergencia de diferentes interpretaciones sobre lo femenino y lo masculino, minimiza al máximo su descripción y voz. De ella sólo sabemos su condición racial y su poca intención de entablar diálogo con Bárbara al creerla poseída por el demonio.
Allí, en el umbral de la puerta, estaba Laura, la vieja criada, la fabulosa criada... Allí esta- ba, de pie en la escalinata, tan rígida y tan negra que, a no ser por el ligero temblor de su rosario de semillas de aguaribay, se la hubiera confundido con uno de los figurones de talla- da cantería que, enmohecidos por la humedad, sostenían angustiosamente los arquitrabes del portón (58).

Destaca además que, al partir hacia la ciudad, la protagonista nunca se pregunta por Laura y parece marcharse dejando a su sirvienta como una parte más del jardín. Si de lo que se trata es de ver en la obra de Loynaz un ejemplo representativo de la inclusión de la mujer, como intentan hacer las relecturas antes mencionadas, tendríamos que aclarar que sólo se trata de un tipo de mujer. Bárbara, blanca, es la única con voz ampliada dentro de la novela. Es entonces necesario un análisis que tenga en cuenta las múltiples dominaciones que se solapan y hacen que los sujetos ocupen diferentes lugares de poder (Meza Mejía, 2018) o subordinación.

\section{Conclusiones}

Esta última acotación invita a la reflexividad dentro de las investigaciones. Al respecto, es necesario decir que, como académicas, estamos en la obligación de evitar generalizaciones sobre el papel de la mujer, de allí los cuestionamientos que realizamos en este artículo. Aun así, es preciso denotar que el margen de acción para la crítica cada vez es menos amplio dadas las condiciones de tiempo y forma que se imponen a los 
investigadores. En ese sentido, este ensayo es un intento por ampliar los márgenes de interpretación ya realizados por los expertos.

Somos conscientes de que este texto ha sido escrito desde y para la academia. Aun así, es un primer paso para transitar hacia un pensar orgánico que sea con y para los sujetos. Por lo tanto, constituye más un ensayo de deconstrucción y tránsito que de solución definitiva y construcción con el otro.

Realizar análisis literarios parece ser una labor menos comprometida que múltiples iniciativas de investigación colaborativa. Sin embargo, creemos que es un esfuerzo acotado y atemperado a las condiciones que se imponen al ámbito científico en el que concurrimos. Es una apuesta ética optar por textos sobre sujetos a la hora de hacer investigación y producción de artículos académicos, puesto que, a pesar de las posibilidades de co-labor, es necesario contar con un tiempo de inmersión y reflexión conjunta con las comunidades que, desafortunadamente, no es posible en los marcos institucionales, de manera que irrumpir en un grupo de investigación puede generar más dificultades que aportes.

Cabe entonces preguntarse por la posibilidad de transitar hacia nuestra participación como sujetos políticos más que como científicos sociales, de suerte que los aportes a los sujetos de estudio pasen a convertirse en acciones y reflexiones para entendernos a nosotros mismos mediante y con los otros. Por ahora, vemos en la literatura el potencial para desestructurar las divisiones entre ciencia y saber y abonar el terreno, como hace Loynaz, hacia una crítica compleja y multiforme de la realidad.

\section{REFERENCIAS}

Barthes, Roland (1984), "La muerte del autor", en El susurro del lenguaje, París, Editions du Seuil, pp. 65-72.

Caballero, Rufo (2005), Hongo fino. La modernidad en Jardín, de Dulce María Loynaz. Imantación y delirio, Santiago de Cuba, Editorial Oriente.
Camejo Ochoa, Hilvimar (2014), "Las ciencias sociales en América Latina: un análisis desde el enfoque de Walter Mignolo”, Sociológica, año 29, núm. 81, pp. 293-292.

Fernández López, Justo (comp.) (2000), "Novela regionalista o de la tierra", en Hispanoteca. Lengua y cultura hispanas [entrada en un sitio web], disponible en: http://www.hispanoteca.eu/Literatura\%20LA/Novela\%20regionalista\%20 o\%20de\%20la\%20tierra.htm

Hall, Stuart (2006), "Estudios Culturales: dos paradigmas", Revista Colombiana de Sociología, núm. 27, pp. 233-254.

Linares, Raúl Fernando (2011), “Apuntes para la re-construcciónde una sociología de la literatura”, Culturales, vol. VII, núm. 13, pp. 115-144.

Loynaz, Dulce María (1992), Jardín, Barcelona, Seix Banal.

Lugones, María (2005), "Multiculturalismo radical y feminismos de mujeres de color", Revista Internacional de Filosofía Política, núm. 25, pp. 61-76.

Magnarelli, Sharon (1996), "La mujer y la naturaleza en La voragine: a imagen y semejanza del hombre", en Saul Sosnowski (ed.), Lectura crítica de la lectura americana. La formación de las culturas nacionales, Caracas, Biblioteca Ayacucho, pp. 506-533.

Márquez Rodríguez, Alexis (2002), "Narrativa hispanoamericana del siglo XX", Hispanista, vol. II, núm. 8, s/n.

Martínez Malo, Aldo (1999), Confesiones de Dulce María Loynaz, La Habana, José Martí Pub House.

Meza Mejía, Sofía A. (2018), "Interseccionalidad”, en José Ramírez Plascencia (coord.), Conceptos claves en ciencias sociales. Definición y aplicaciones, Guadalajara, Universidad de Guadalajara, pp. 235-256.

Millares, Selena (1993), "Dulce María Loynaz, en el tallo de los vientos", en De Vallejo a Gelman: un siglo de poetas para Hispanoamérica, Murcia, Cuadernos de América sin nombre, pp. 55-70.

Mondragón Espinoza, Blanca Aurora (2008), “'La bella durmiente del bosque', una historia revisitada. Intertextualidad en Jardín de Dulce María Loynaz", La Colmena, núm. 59 , pp. 20-33.

Oyarzún, Luis (2000), "Hombre, sociedad y naturaleza en la literatura latinoamericana", Revista de Filosofía de la Universidad de Chile, vol. 6, núm. 1, pp. 26-30.

Polo García, Victorino (1982), Voces de Hispanoamérica. Estética y telurismo en la literatura, Murcia, Planetaria.

Smith, Verity (1993), “'Eva sin paraíso': una relectura feminista de Jardín de Dulce María Loynaz", Nueva Revista de Filología Hispánica, t. 41, núm. 1, pp. 263-277.

Stavenhagen, Rodolfo (1981), "Siete tesis equivocadas sobre América Latina”, en Sociologia y subdesarrollo, México, Nuestro Tiempo, pp. 15-84.
Laura Carolina Castañeda Sua. Politóloga por la Universidad Nacional de Colombia (UNAL), Colombia. Estudiante de la Maestría en Ciencias Sociales de la Universidad de Guadalajara (UDG), México. Sus intereses académicos son los estudios decoloniales, el género, la ecología política y la literatura. 